Tropical Journal of Pharmaceutical Research, June 2002; 1 (1): 15-22

(C) Pharmacotherapy Group, Faculty of Pharmacy, University of Benin, Benin City, Nigeria.

All rights reserved.

Available online at http://www.tjpr.freehosting.net

Research Article

\title{
Novel colorimetric assay of mefenamic acid using 4- amino-3,5-dinitrobenzoic acid (ADBA)
}

\author{
SO Idowu ${ }^{\dagger}$, SC Tambo, AO Adegoke and AA Olaniyi
}

Department of Pharmaceutical Chemistry, Faculty of Pharmacy, University of Ibadan, Ibadan.

\begin{abstract}
Purpose: To develop a novel colorimetric assay method for mefenamic acid capsules.

Method: The new method (aromatic ring derivatization technique) is based on a diazo coupling reaction using diazotized 4-amino-3,5-dinitrobenzoic acid (ADBA) as a chromogenic derivatizing reagent.

Result: Optimization studies showed that the coupling reaction is very fast and completed in less than 1 minute. A 1:1 drug to reagent stoichiometric ratio was obtained for the azo dye formed. The azo adduct formed exhibits bathochromic shift with absorption maximum $\left(\lambda_{\max }\right)$ at $490 \mathrm{~nm}$, which was selected as the analytical wavelength. Lower limit of quantitation of mefenamic acid was $1 \mathrm{ug} / \mathrm{ml}$. The assays were linear over the concentration range of 1 - 6 $\mu \mathrm{g} / \mathrm{ml}$ and reproducible. This new method has been successfully applied in the assay of mefenamic acid capsules with accuracy similar to the official (B.P) titrimetric method of assay $(p>0.05)$ and has the advantages of speed, high sensitivity, lower limit of detection and can be automated.

Conclusion: The method developed could find application in in-process quality control of mefenamic acid capsules.
\end{abstract}

Keywords: Mefenamic acid assay, 4-amino-3,5-dinitrobenzoic acid, colorimetry, diazotization.

†To whom correspondence should be addressed. E-mail: olakunleid@yahoo.com Tel: +234 8023094981 


\section{Idowu et al}

\section{Introduction}

Mefenamic acid is a non-steroidal antiinflammatory drug (NSAID) which has analgesic, anti-inflammatory and antipyretic actions. It is been used for the relief of mild to moderate pain. It is also indicated for the treatment of rheumatoid arthritis ${ }^{1}$, primary dysmenorrhea $^{2}$ and periodontitis ${ }^{3}$. The official method for the assay of the pure drug and the capsules is titrimetry, using sodium hydroxide as titrant and phenol red as indicator ${ }^{4,5}$. A variety of methods have been used for the determination of the drug in biological fluids. Determination in the serum has been carried out with high performance liquid chromatography (HPLC) with UV detection and spectrofluorimetry ${ }^{7}$. Determination in the blood has been carried out with HPLC with photodiode array detector and gas chromatography (GC) with flame ionisation detector $^{8}$. Determination in urine was reportedly done by HPLC ${ }^{9}$.

Trends in pharmaceutical analysis and compendial assay methods reflect increasing replacement of time-honoured classical methods of analysis with modern, more sophisticated instrumental methods, especially HPLC. However, the high acquisition and maintenance cost of this equipment makes adoption of assay methods based on them rather inconvenient, especially in poor-resource economies. This often warrants the use of an alternative method. The British Pharmacopoeia gives allowance for the use of an alternative method for any test or assay, if it is known that the method used will be of equivalent accuracy with the prescribed assay method ${ }^{4}$.

Colorimetry as an analytical technique became obsolete with the advent of scanning spectrophotometers. By means of the monochromator, discrete wavelength interval in the ultraviolet, visible and near infra-red region can be isolated easily on spectrophotometers. However, the simpler architecture of digital colorimeters using built- in gelatin filters and drop-in interference filters means initial acquisition and maintenance costs are much lower. Vastly improved electronics, integrated circuitry and microprocessor technology incorporated into the design of modern colorimeters now produce two to three decimal place resolution. In addition, they have interfacing capabilities with computers. These attractive features led to a resurgence of interest in colorimetric assay of pharmaceuticals. Modern colorimetry provides a unique combination of high technology and low cost in analytical methodology; it shares the typical merits of instrumental methods, especially, faster handling of large sample throughput ${ }^{10}$.

In this paper, we report a novel colorimetric assay of mefenamic acid in pharmaceutical dosage forms using a newly developed chromogenic derivatizing reagent. The reagent, diazotized 4-amino-3,5dinitrobenzoic acid (ADBA), is a dinitrophenyl arenediazonium ion, which functions via an aromatic ring derivatization technique based on diazo coupling reaction ${ }^{11-13}$.

\section{Experimental}

\section{Chemicals and Reagents}

Ponstan capsules (Parke-Davis, Istanbul,Turkey), Mefacap® capsules (Malaysia Pharmaceutical Factory, Malaysia), mefenamic acid crystals (isolated and purified in our laboratory), ethanol, ethyl acetate, glacial acetic acid, concentrated sulphuric acid, sodium nitrite, potassium hydrogen phthalate, sodium hydroxide pellets, phenol red crystals, dried magnesium sulphate (all are analytical reagents from $\mathrm{BDH}, \mathrm{UK}$ ).

\section{Equipment}

UV/visible spectrophotometer (Unicam Aurora, Helios Scan, software v 1.1), digital colorimeter, model 6051 (Jenway, U.K), Mettler balance H80 (U.K.), ultrasonic bath (Langford, U.K). 


\section{Idowu et al}

\section{Method}

The solution of ADBA in concentrated sulphuric acid was diazotized with sodium nitrite as previously reported ${ }^{11}$. Sodium hydroxide $(0.1 \mathrm{M})$ was prepared and standardized as previously described ${ }^{5}$. Mefenamic acid stock solution was ma de by dissolving mefenamic acid crystals $(10 \mathrm{mg})$ in hot (about $\left.70{ }^{\circ} \mathrm{C}\right)$ glacial acetic acid $(6 \mathrm{ml})$ with stirring. The solution was allowed to cool and the volume was then made up to $10 \mathrm{ml}$ in a volumetric flask by adding fresh glacial acetic acid.

In order to determine a suitable analytical wavelength for the reaction product, two critical response parameters (temperature and reaction time) were optimized using the method of steepest ascent ${ }^{14}$. Aliquot of mefenamic acid stock solution $(50 \mu \mathrm{l})$ was added to the reagent solution $(500 \mu \mathrm{l})$ in a test tube and the reaction mixture was mixed in a vortex mixer for $10 \mathrm{sec}$ followed by incubation in turn at $30^{\circ} \mathrm{C}$ and $50^{\circ} \mathrm{C}$ for 5 minutes and 20 minutes, respectively. Similar experiments were performed at $60{ }^{\circ} \mathrm{C}$ and $80^{\circ} \mathrm{C}$. Each determination was done in duplicate. The reaction was terminated by addition of ice-cold water $(5 \mathrm{ml})$ to the reaction mixture kept in icebath. Aqueous solution was extracted with ethyl acetate $(10 \mathrm{ml})$ and kept in a vial wrapped with aluminium foil. A blank reagent solution was prepared in a similar way but replacing the stock solution with glacial acetic acid. The absorption spectrum of the reaction mixture extract was determined against the absorption spectrum of the blank reagent extract using the UV/visible spectrophotometer and the optimal absorption wavelength, $490 \mathrm{~nm}$, was selected for sample analysis.

The optimal reaction time was determined by adding aliquot of mefenamic acid stock solution $(50 \mu \mathrm{l})$ in turn to the reagent solution $(500 \mu \mathrm{l})$ in six tubes. Coupling reaction was carried out by incubation at $30^{\circ} \mathrm{C}$ for $0,1,2,3$, 4 and 5 min. Ethyl acetate extracts of the reaction mixtures was prepared as usual after each reaction time and the absorbance was measured at $490 \mathrm{~nm}$ on the digital colorimeter. An optimal reaction time was then determined as the time corresponding to the maximal absorption of the samples. All determinations were done in duplicate.

The best extraction method was determined by extracting the reaction mixture $(50 \mu \mathrm{l}$ of mefenamic acid stock solution and $500 \mu \mathrm{l}$ of reagent solution, mixed for 10 seconds followed by mixing with $5 \mathrm{ml}$ of ice-cold water to terminate the reaction) with ethyl acetate $(10 \mathrm{ml}$ or $3 \mathrm{ml}$ in 2 portions followed by $4 \mathrm{ml}$ ). Combined extracts of ethyl acetate were dried over anhydrous magnesium sulphate and then filtered in a glass pasteur pipette plugged with cotton wool into a $10 \mathrm{ml}$ volumetric flask. The volume was made up with fresh ethyl acetate and absorbance of the sample determined at $490 \mathrm{~nm}$. This procedure was carried out in triplicates.

\section{Photostability of the azo adduct in ethyl acetate}

Standard test solutions containing $5 \mu \mathrm{g} / \mathrm{ml}$ were prepared in six sample vials. Three of the vials were wrapped with aluminium foil, while the other three were left unwrapped. Both sets were kept on the laboratory bench. The absorbance reading of the extracts at $490 \mathrm{~nm}$ were taken at 30 minutes interval for a period of three hours.

Stoichiometric ratio of drug-reagent adduct formation

Equimolar solutions $\left(9.18 \times 10^{-4} \mathrm{M}\right)$ of the reagent and the drug stock solution were prepared using the procedures described above. In five different test tubes, 0, 2.5, 5.0, 7.5 and $1.0 \mathrm{ml}$ of the reagent solution were added, respectively. Each tube was then made up to $1.0 \mathrm{ml}$ with the drug stock solution. A series of blank determination were carried out in which the volume of the drug stock solution was replaced with glacial acetic 


\section{Idowu et al}

acid. The mixture was mixed in a vortex mixer for $10 \mathrm{sec}$ and the extraction carried out afterwards as usual. The absorbance was measured at $490 \mathrm{~nm}$ against the blank and the absorbance values obtained were plotted against the mole fraction of the reagent solution. Each determination was carried out in duplicate.

\section{Assay of dosage form}

The amount of mixed contents of 20 capsules equivalent to $10 \mathrm{mg}$ of mefenamic acid were weighed and dissolved in hot (about $70{ }^{\circ} \mathrm{C}$ ) glacial acetic acid and mixed in an ultrasonicator for $5 \mathrm{~min}$. The solution was allowed to cool and filtered through a cotton wool plug into a $10 \mathrm{ml}$ volumetric flask. The volume was then made up with fresh glacial acetic acid, rinsing the filter aid in the process. An aliquot of the drug stock solution $(50 \mu \mathrm{l})$ was added to the reagent solution $(500 \mu \mathrm{l})$. The mixture was then mixed in a vortex mixer for $10 \mathrm{sec}$ and extracted with $10 \mathrm{ml}$ of ethylacetate as described above and the absorbance measured at $490 \mathrm{~nm}$. Content of the mefenamic acid capsules were then determined by interpolation from calibration lines. The sample analysis was repeated using the B. $P^{5}$ procedure. Two formulations of mefenamic acid (Ponstan $^{\circledR}$ and Mefacap ${ }^{\circledR}$ ) were used in the assays.

The assay results using the two methods (the method being reported and the B.P. method) were compared using the Student's t-test. A2tailed probability values ( $p$-value) less than or equal to 0.05 at $95 \%$ confidence interval was considered to be significant.

\section{Validation of methods.}

Calibration lines using standard solutions of 0 , $2.5,5,10,20,40$ and $80 \mu \mathrm{g} / \mathrm{ml}$ mefenamic acid were carried out using the optimal analytical conditions as described above. Linear regression analysis was used to calculate the slope, intercept and the correlation coefficients $\left(r^{2}\right)$ of each calibration line. The assay precision and accuracy were determined as previously described ${ }^{14}$. The limit of detection was computed as previously described as the analyte concentration giving a signal equal to the blank signal plus three standard deviations of the blank ${ }^{15}$.

\section{Results}

The spectrum of mefenamic acid in ethyl acetate showed two absorption maxima at $280 \mathrm{~nm}$ and $350 \mathrm{~nm}$ (Figure 1). Coupling reaction of mefenamic acid and reagent solution formed a reddish-pink adduct under all coupling conditions tried and the spectrum of the adduct indicated bathochromic shift,

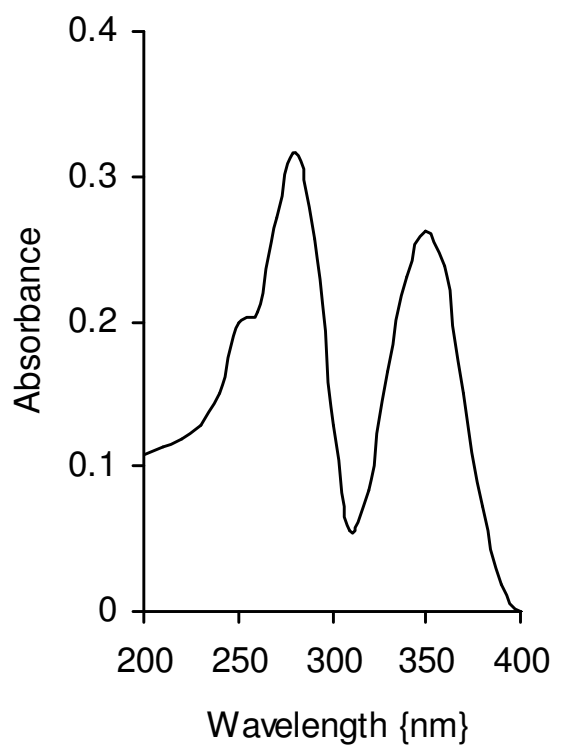

Figure 1: Absorption spectrum of mefenamic acid in ethyl acetate

with an optimal peak at $490 \mathrm{~nm}$. The blank reagent also has peaks at $260 \mathrm{~nm}, 340 \mathrm{~nm}$ and $430 \mathrm{~nm}$ (Figure 2). Absorbance of the adduct was highest at $30^{\circ} \mathrm{C}$ and $5 \mathrm{~min}$ reaction time (Figure 3 ). There was no significant change in absorbance over the reaction time investigated (Figure 4).

Comparison of the single and multiple extraction process revealed that multiple 


\section{Idowu et al}

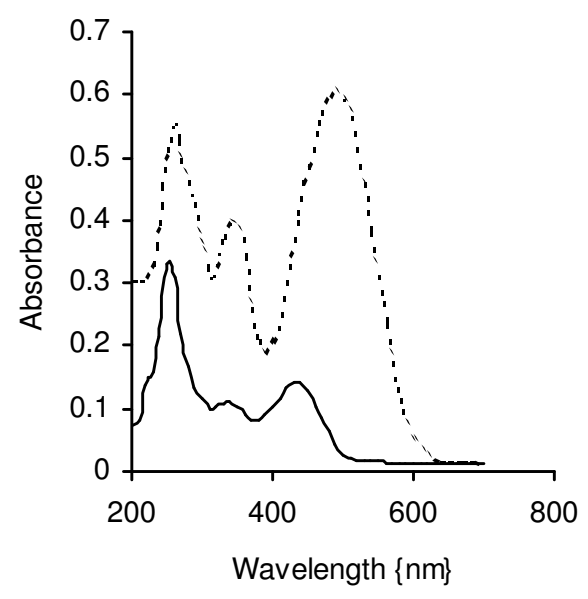

Figure 2: Overlaid absorption spectra of blank reagent $(-)$ and coupling reaction mixture (test, ------) extract

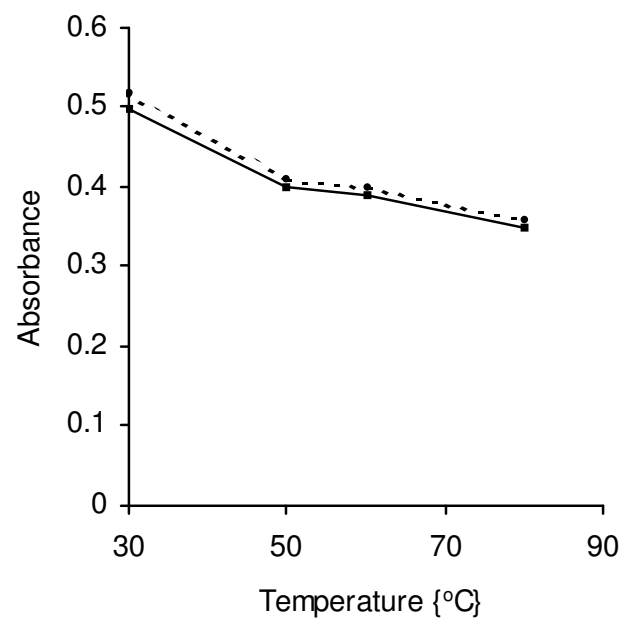

Figure 3: Optimization of coupling reaction temperature (---, $5 \mathrm{~min} ;-20 \mathrm{~min})$

extraction offered no advantage as the extraction of the adduct with a single lot of 10 $\mathrm{ml}$ ethyl acetate gave mean absorbance valueof $0.34 \pm 0.01$ while the multiple extraction process gave mean absorbance value of $0.33 \pm 0.01$ at $490 \mathrm{~nm}$. Maximum absorbance of the adduct was obtained at a mole fraction of 0.5 for the reagent solution (Figure 5) and the absorbance was found to decrease at a lower or higher mole fractions.

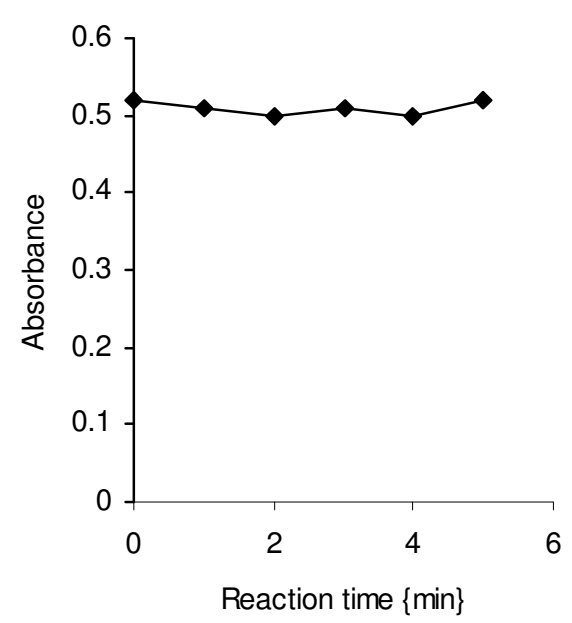

Figure 4: Optimization of reaction time at $30^{\circ} \mathrm{C}$

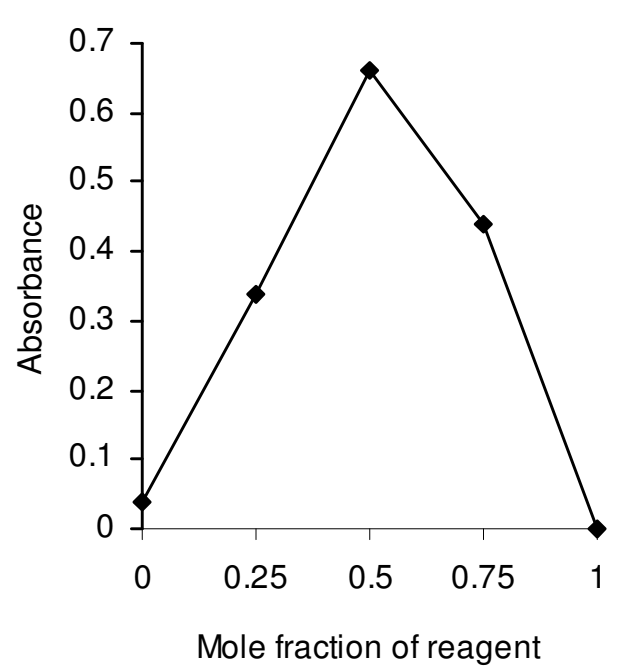

Figure 5: Determination of stoichiometric ratio of drugreagent adduct

The azo adduct formed by the coupling reaction was found to be stable to light over a period of $3 \mathrm{~h}$.

Comparative analysis of two brands of mefenamic acid capsules by the official titrimetric method and the new method is shown in Table 1. There was no significant 
Table 1: Assay of mefenamic acid capsules by official method and the new method

\begin{tabular}{ccc}
\hline & \multicolumn{2}{c}{ Mean \pm S.D } \\
Dosage form brand & \multicolumn{2}{c}{ B.P } \\
\cline { 2 - 3 } & ADBA $^{*}$ & B. \\
\hline Ponstan $^{\circledR}$ & $101.70 \pm 1.43$ & $101.80 \pm 0.12$ \\
Mefacap $^{\circledR}$ & $100.16 \pm 1.44$ & $100.43 \pm 0.15$ \\
\hline
\end{tabular}

${ }^{*} n=8,=n=5$, Official range $=95.0-105 \%(B . P$. 1998), $p<0.05$ is taken as significant.

$A D B A=$ New colorimetric assay method; B.P. $=$ Official titrimetric method.

difference between the titrimetric method of B.P. and the method being reported $(p>$ 0.05).

Assay regression equation for the calibration line under the optimal conditions is $y=$ $0.0646 x+0.024$ with $r^{2}$ of 0.9875 . The limit of detection was determined as $0.96 \mu \mathrm{g} / \mathrm{ml}$. The overall recovery of extracted samples is $101.88 \pm 1.37 \%$ and the coefficient of variation is $1.36 \%$ (Table 2). Repeatability of extraction was found to be better for the larger analyte ( 6 $\mu \mathrm{g} / \mathrm{ml}$ ) when compared to lower quantities of the analytes.

\section{Discussion}

Diazo coupling of mefenamic acid with the reagent produced bathochromic shift in the absorption spectm of the reaction mixtures (Figure 2) relative to the absorption spectrum of mefenamic acid alone (Figure 1). This is

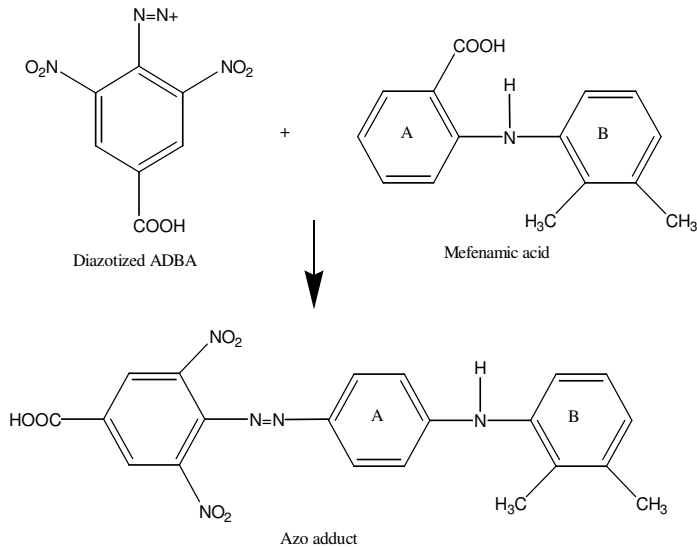

Scheme 1: Coupling reaction between diazotized ADBA and mefenamic acid

indicative of the formation of an azo dye (Scheme 1). Mefenamic acid has two aromatic rings ( $\mathrm{A}$ and $\mathrm{B}$ ) joined by an amino bridge. The para position to the amino group is favoured for coupling on both rings, considering the combined directing influence of the ring substituents toward electrophilic aromatic substitution reaction. However, the para position on ring $B$ is more sterically hindered thus making ring $A$ the most likely point of attack. The very fast reaction observed is due to the strong ring activating effect of the amino group and the meta directing influence of the carboxyl group. Both substituents favour the same point of attack. The peak at $490 \mathrm{~nm}$ was selected as the analytical wavelength because at this wavelength, the difference in absorptivity between the blank reagent and the adduct is

Table 2: Three-day assessment of accuracy and precision of the new method of assay of mefenamic acid

\begin{tabular}{ccccccc}
\hline $\begin{array}{c}\text { Concentration } \\
(\mu \mathrm{g} / \mathrm{ml})\end{array}$ & \multicolumn{2}{c}{ Day 1} & \multicolumn{2}{c}{ Day 2} & \multicolumn{2}{c}{ Day 3} \\
\cline { 2 - 7 } & Mean $\pm \mathrm{s} . \mathrm{d}^{*}$ & $\mathrm{RSD}$ & Mean $\pm \mathrm{s} . \mathrm{d}^{*}$ & $\mathrm{RSD}$ & Mean $\pm \mathrm{s} . \mathrm{d}^{*}$ & $\mathrm{RSD}$ \\
\hline 2.0 & $100.10 \pm 4.47$ & 4.47 & $102.68 \pm 4.47$ & 4.35 & $102.68 \pm 4.47$ & 4.35 \\
4.0 & $100.36 \pm 2.23$ & 2.22 & $101.65 \pm 2.34$ & 2.3 & $101.65 \pm 2.33$ & 2.19 \\
6.0 & $100.45 \pm 1.49$ & 1.48 & $99.59 \pm 2.58$ & 2.59 & $98.73 \pm 1.49$ & 1.51 \\
\hline
\end{tabular}

${ }^{*} \mathrm{n}=9$; Regression equation: $Y=0.0646 x+0.024\left(r^{2}=0.9875\right)$; Between-day statistics: $=100.88 \pm 1.37 \%$ (mean \pm s.e.m) RSD (of s.e.m) $=1.36 \%$ 
maximal ${ }^{16}$. Incubation at elevated temperature was found to be unnecessary, because thermal degradation led to a lower detector response at temperatures higher than $30^{\circ} \mathrm{C}$ (Figure 3). Incubation at even $30^{\circ} \mathrm{C}$ for any length of time was shown to be unnecessary. The reaction is shown to be so fast as to be completed in less than $1 \mathrm{~min}$. The absorbance of the test extract prepared immediately after mixing in a vortex mixer for 10 seconds is the same as the absorbance after $5 \mathrm{~min}$ of incubation at $30^{\circ} \mathrm{C}$ (Figure 4).

In theory, multiple extraction with organic solvents guarantees complete extraction than single extraction procedure. However, because of the low concentrations involved (microgram quantities) and the partition coefficient of the adduct in the solvent system employed, single extraction is as efficient as multiple extraction. In addition, time is gained in the overall analysis with single extraction.

For a constant total concentration of two interacting species, the complex is at its greatest concentration at a point where the two species are combined in the ratio in which they occur in the complex ${ }^{17}$. The plot of absorbance against the mole fraction of the reagent exhibited a change of slope with a maximum absorbance at a mole fraction of 0.5 and decreasing absorption as the value of the mole fraction deviate from 0.5 (Figure 5). The implication of this is that better absorbance of the adduct will be obtained at equimolar concentrations of the mefenamic acid sample and the reagent. This also signifies that the optimal coupling reaction conditions routinely employed forms the azo dye by a 1:1 drug to reagent stoichiometric ratio as depicted in Scheme 1.

Classical methods are typically more precise than instrumental methods ${ }^{10}$, as such, the official titrimetric method was more precise than the colorimetric method reported here. However, the new method is of equivalent accuracy to the official method (Table 2).

\section{Conclusion}

A novel colorimetric assay method for mefenamic acid capsules based on a very fast diazo coupling reaction of the drug with diazotized ADBA has been developed. The new method was shown to be of equivalent accuracy with the official (B.P) titrimetric method. It has the advantages of speed, high sensitivity and lower limit of detection. The new method can also be easily automated by interfacing the digital colorimeter with computers. It could find application in inprocess quality control of mefenamic acid capsules.

\section{References}

1. Martindale, The Extra Pharmacopoeia, $28^{\text {th }}$ ed. The Pharmaceutical press, London. 1982:pp 262263.

2. Zhang WY and Li Wan PA. Efficacy of minor analgesics in primary dysmenorrhea: a systematic review. British Journal of Obstetrics and Gynaecology, 1998;105(7):780-789.

3. Cory $D$ and Moran J. Assessment of acrylic bone cement as a local delivery vehicle for the appliation of non-steroidal anti-inflammatory drugs. Biomaterials 19(14):1295-1301.

4. British Pharmacopoeia. Volume I. Her Majesty's Stationery Office, London., 1998:pp 849-851, p 11.

5. British Pharmacopoeia. Volume 2. Her Majesty's Stationery Office, London., 1998:p 1793.

6. Rawasheh NM, Najib NM and Jalal IM. Comparative bioavailability of two capsule formations of mefenamic aacid. International Journal of Clinical Pharmacology and Therapeutics, 1997:35(8):329-333.

7. Ionnaou PC, Rusakova NV, Andrikopoulou DA, Glynou KM and Tzompanaki GM. Spectrofluorimetry determination of anthranilic acid derivatives based on terbium sensitized fluorescence. Analyst,1998:123(12):2839-2843.

8. Lo DS, Chao TC, Ng-Ong SE, Yao YJ and Koh TH. Acid and neutral drug screen in blood with quantitation using microbore high-performance liquid chromatography-diode array detection and capillary gas chromatography-flame ionization detection. Forensic Science International, 1997:90(3):205-214.

9. Hirai $\mathrm{T}$, Metsumoto $\mathrm{S}$ and Kishi I. Simultaneous analysis of several non-steroidal antiinflammatory drugs in human urine by high performance liquid chromatography with normal solid-phase extraction. Journal of Chromatography B, Biomedical Sciences 


\section{Idowu et al}

\&Applications, 1997:692(2)375-388.

10. Willard HH, Meritt LL (Jr), Dean JA and Settle, FA (Jr). An Introduction to instrumental methods of analysis. In: Instrumental Methods of Analysis, ed. 7, Belmont California, USA, Wordsworth Publishing Company, 1988: pp. 6-7.

11. Idowu SO. Development and evaluation of 4-amino3,5-dinitrobenzoic acid (ADBA) as a novel derivatizing reagent. Ph.D. Thesis, University of lbadan, 1998

12 Idowu SO, Fasanmade AA, Fatoki SA and Olaniyi AA. A novel approach to spectrophotometric determination of ephedrine via the 4-amino-3,5dinitrobenzoic acid (ADBA) azo derivative. Journal of Pharmaceutical Research and Development 1997:2(2):14-30.

13. Idowu $S O$ and Olaniyi AA. Evaluation of diazotized 4-amino-3,5-dinitrobenzoic acid (ADBA) as a new derivatizing reagent. African Journal of Medicine and Medical Sciences. 2001:30:217220.
14. Erah PO, Barrett DA and Shaw PN. Reversedphase high-performance chromatographic assay methods for the analysis of a range of penicillins in in vitrro permeability studies. $J$. Chromatogr B, 1998:63-69

15. Miller JC and Miller JN. Method of Steepest Ascent. In: Statistics for Analytical Chemistry, ed. 3, Prentice Hall London, Ellis Horwood PTR, 1993:pp. 188-190, p 115, pp 103-104.

16. Willard HH, Meritt LL (Jr), Dean JA and Settle, FA (Jr). Ultraviolet and Visible Absorption $m \quad \mathrm{e} \quad \mathrm{t} \quad \mathrm{h} \quad \mathrm{o} \quad \mathrm{d} \quad \mathrm{s}$. In: Instrumental Methods of Analysis, ed 7, Belmont, California, USA, Wordsworth Publishing Company, 1988:pp. 169-173.

17. Martin A, Swarbrick $J$ and Cammarata A. Complexation and protein binding. In: Physical Pharmacy:physical chemical principles in the pharmaceutical sciences, ed. 3, Lea \& Febiger, Philadelphia, 1983:pp. 325-326. 